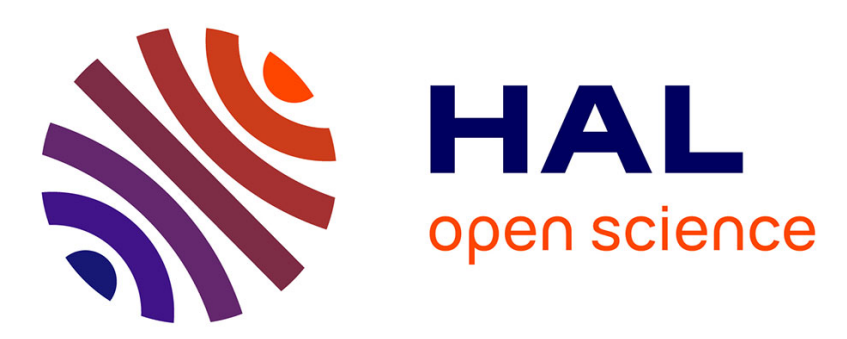

\title{
Processus et bilan des flux hydriques d'un bassin versant de milieu tropical de socle au Bénin (Donga, haut Ouémé)
}

\author{
Bamory Kamagate, Luc Seguis, Guillaume Favreau, J.L. Seidel, Marc
}

Descloitres, P. Affaton

\section{To cite this version:}

Bamory Kamagate, Luc Seguis, Guillaume Favreau, J.L. Seidel, Marc Descloitres, et al.. Processus et bilan des flux hydriques d'un bassin versant de milieu tropical de socle au Bénin (Donga, haut Ouémé). Comptes Rendus Géoscience, 2007, 339 (6), pp.418-429. 10.1016/j.crte.2007.04.003 . ird-00165994

\section{HAL Id: ird-00165994 https://hal.ird.fr/ird-00165994}

Submitted on 31 Jul 2007

HAL is a multi-disciplinary open access archive for the deposit and dissemination of scientific research documents, whether they are published or not. The documents may come from teaching and research institutions in France or abroad, or from public or private research centers.
L'archive ouverte pluridisciplinaire HAL, est destinée au dépôt et à la diffusion de documents scientifiques de niveau recherche, publiés ou non, émanant des établissements d'enseignement et de recherche français ou étrangers, des laboratoires publics ou privés. 
Processus et bilan des flux hydriques d'un bassin versant de milieu tropical de socle au Bénin (Donga, haut Ouémé)

Bamory Kamagaté, Luc Séguis, Guillaume Favreau, Jean-Luc Seidel, Marc Descloitres, Pascal Affaton

Comptes Rendus Geoscience

http://france.elsevier.com/direct/CRAS2A

Volume 339, Numéro 6, Juin 2007

pp. $418-429$

also available online at www.sciencedirect.com

«Avis : Ce texte est la version de l'auteur d'une oeuvre acceptée en vue de sa publication dans les Comptes-Rendus Geoscience. II est dès lors possible que les modifications résultant du processus de publication, et notamment de l'examen par les pairs, la révision, l'édition, les corrections, la mise en page structurelle et les autres dispositifs de contrôle de qualité ne soient pas reprises dans le présent document. II est possible que des modifications aient été apportées à la présente œuvre après qu'elle ait été présentée en vue de sa publication. Une version définitive a par la suite été publiée dans Comptes Rendus Geoscience, vol. 339, no. 6, Juin 2007, doi : 10.1016/j.crte.2007.04.003 » 
Comptes Rendus Geoscience

Hydrologie-Hydrogéologie / Hydrology-Hydrogeology

Processus et bilan des flux hydriques d'un bassin versant de milieu tropical de socle au Bénin (Donga, haut Ouémé)

Hydrological processes and water balance of a tropical crystalline bedrock catchment in Benin (Donga, upper Ouémé River)

Bamory Kamagaté ${ }^{1}$, Luc Séguis ${ }^{1}$, Guillaume Favreau, ${ }^{1,{ }^{*}}$, Jean-Luc Seidel ${ }^{1}$, Marc Descloitres $^{2}$ et Pascal Affaton ${ }^{3}$

1 UMR HydroSciences (IRD, CNRS, UMI, UMII), Université de Montpellier II, place E. Bataillon, CC MSE, 34095 Montpellier cedex 5, France

* auteur correspondant / corresponding author. Tél : (+216) 71750 009; Fax : (+216) 71750 254. Guillaume.Favreau@ird.fr

${ }^{2}$ IRD, UMR LTHE, BP 53, 38041 Grenoble cedex 09, France.

${ }^{3}$ Université Aix-Marseille, CEREGE, Europôle de l'Arbois, BP 80, 13545, Aix-en-Provence cedex 4, France. 


\title{
Résumé
}

L'étude d'un bassin versant de $586 \mathrm{~km}^{2}$ au Bénin (Afrique de l'ouest) par des suivis hydrodynamiques et hydrochimiques, ainsi que par reconnaissance géophysique de sub-surface, a permis de déterminer les processus et le bilan des flux hydriques sur deux années à pluviométrie contrastée. La recharge de la nappe phréatique s'effectue par infiltration directe des précipitations et représente de 5 à 24\% de la pluviométrie. L'écoulement à l'exutoire, limité à la saison des pluies ainsi que la minéralisation faible des eaux indiquent des flux nappe - rivière négligeables. Cette faible contribution de la nappe implique que les variations interannuelles du coefficient d'écoulement (de 14\% à 28\%) sont principalement gouvernées par des flux de surface et de sub-surface.

\begin{abstract}
Hydrodynamic, geochemical and sub-surface geophysical investigations, for two consecutive years with contrasting rainfall conditions, were used to characterize the hydrological processes occuring, and the water balance of a $586-\mathrm{km}^{2}$ watershed in Benin (Africa). The water table's monitoring shows that recharge occurs by direct infiltration of rainfall, and represents between 5 to $24 \%$ of the annual rainfall. Both surface water outflow, limited to the rainy season, and the water chemistry indicate a weak groundwater contribution to river discharge. This implies that the calculated variations in annual runoff coefficients (of 14 and 28\%) are mainly governed by surface and subsurface flows.
\end{abstract}

Mots clés : Bénin, aquifère libre, hydrochimie, hydrodynamique, rivière tropicale Key words: Benin, groundwater, hydrochemistry, hydrodynamics, tropical river 


\section{Abridged English version}

\section{Introduction}

In West Africa, the rainfall deficit of about 20 to $30 \%$ observed since the early seventies [18, 21] has led to a higher decrease in river discharge [23]. This implicitly non-linear relationship was mainly studied for large river basins, by indirect or conceptual methods $[23,24]$; the rationale is that lower rainfall resulted in lower groundwater discharge into the rivers. However, studies performed at a finer scale [e.g. 6] suggested more complex surface-subsurface flow processes.

The AMMA project (African Monsoon Multidisciplinary Analysis, http://ammainternational.org) was designed to better understand the African monsoon, and its hydrological impacts. For this goal, surveys have been performed since 2003 in a $586-\mathrm{km}^{2}$ catchment in central Benin (Fig. 1), a key-region for understanding the impact of climate [19], and land-use changes [14] on the regional hydrological cycle.

This paper presents the first 2-year hydrodynamic, geochemical, and subsurface geophysical surveys performed within this basin. A first estimate of the water balance is obtained for these two years, for which contrasting rainfall amounts were recorded.

\section{Study area}

The Donga river (Fig. 1) is an affluent of the Ouémé river (523 km), in central Benin [17]. The tropical climate is characterized by a dry and a rainy season, with a mean annual rainfall measured at Djougou of $1195 \mathrm{~mm}$ (1950-2004). The potential evapotranspiration (ETP, Penman-Monteith [10]) is of $1390 \mathrm{~mm} \mathrm{yr}^{-1}$ (2002-2005).

Altitude ranges from $520 \mathrm{~m}$ to $340 \mathrm{~m}$ for a river slope of $1.7 \mathrm{~m} \mathrm{~km}^{-1}$. Outcroping siliceous rocks are of metamorphic origin $[1,5]$. The weathered bedrock has a thickness of 10 to $25 \mathrm{~m}$ and represents the matrix of the phreatic aquifer. In depth, some faults also contain deeper groundwater, in hydraulic continuity with the overlaying reservoir [16]. The soils are classified as polycyclic ferruginous soils [12], but change in details depending on the topography, rock type or land use [11]. The natural wooded savannah has been largely cleared and most of the land is now 
covered by rain-fed crop fields; a forest gallery of a few tens of metres runs along most of the streams.

The population density is of 30 inhab. $\mathrm{km}^{-2}$, and has increased recently by $4.9 \% \mathrm{yr}^{-1}$ (1992-2002). Most of the domestic water comes from wells scattered in the basin. Our surveys indicate a water use of $21 \mathrm{~L}_{\text {inhab.day }}^{-1}$, a value close to the $17 \mathrm{~L}$ inhab.day ${ }^{-1}$ estimated at a regional scale [15]. Considering the population density, this implies a human-induced discharge from the aquifer close to $0.2 \mathrm{~mm} \mathrm{yr}^{-1}$.

\section{Data}

Electrical resistivity data were obtained in May, 2003, with a view to characterize the weathered substratum; changes were expected for differences in rock type, clay, and/or water content [8]. The electrical tomography method [22] was implemented at a scale of several hundred meters on few representative slopes; a geological survey helped in constraining the interpretation.

Hydrodynamics data records were obtained on 16 rain gauges (each $0.5 \mathrm{~mm}$ ) and 26 wells (each $30 \mathrm{mn}$ or 3 times a day). Additional data from an upstream site were obtained in 2004 from 9 piezometers screened at different depths (2, 10 and 20 m; Fig. 1). For surface waters, three limnimetric records (time steps of 5 or $30 \mathrm{mn}$ ) represent catchments of respectively 13, 105 and $586 \mathrm{~km}^{2}$ (Fig. 1). A differential GPS campaign was also performed to characterize the hydraulic potentials [16].

Hydrochemical data (temperature, $\mathrm{pH}, \mathrm{EC} 25^{\circ} \mathrm{C}$ ) were obtained monthly or quarterly on 20 wells; some measurements were also performed on bulk rainfall events. Measurements on surface waters were made for springs, tributaries and on the Donga River. The limnimetric station $\mathrm{K}$ was equipped in June 2003 with an EC recorder (time step of $30 \mathrm{~min}$ ). Other EC recorders with the same time step were settled in April 2004 on the nested piezometers on the Ara sub-catchement. Chemical analysis $\left(\mathrm{Ca}^{2+}, \mathrm{Mg}^{2+}, \mathrm{Na}^{+}, \mathrm{K}^{+}\right.$for cations, $\mathrm{HCO}_{3}^{-}, \mathrm{SO}_{4}{ }^{2-}, \mathrm{NO}_{3}{ }^{-}, \mathrm{Cl}^{-}$for anions) were performed on samples from rain water (18 analyses), ground water (23 wells and 15 boreholes), seasonal springs (8 analyses) and water from the Donga River (a dozen of analyses for each station); in addition, three main flood events were surveyed by sequential sampling in 2003. All the samples were filtered in the field and subsequently analysed by capillary electrophoresis (cations and $\mathrm{SO}_{4}{ }^{2-}, \mathrm{NO}_{3}{ }^{-}$ , $\left.\mathrm{Cl}^{-}\right)$or acidic titration $\left(\mathrm{HCO}_{3}^{-}\right)$, with a precision of about $5 \%$. 


\section{Results}

\subsection{Subsurface geophysics}

A typical example of the resistivity profiles performed within the limits of the basin $[8,16]$ is displayed in Figure 2; it reveals an important heterogeneity of the resistivity at the hectometric scale. The bedrock lies from 15 to $25 \mathrm{~m}$ below the soil surface. Clayey materials (electrically conductive) and/or indurated covers, or quartzite outcrops (both resistive) occur at surface and may locally outcrop; the clayey layers act as preferential places for the formation of perched groundwaters.

\subsection{Hydrodynamics}

The total rainfall amount is of 25\% lower in 2004 than in 2003 but the difference is even higher for total runoff, with a decrease of $64 \%$ (Table 1). The river flow shows rapid changes, with daily discharge reaching a maximum of $140 \mathrm{~m}^{3} \mathrm{~s}^{-1}$ at the basin outlet; at the seasonal scale, the discharge period is shorter in 2004 than in 2003, under the influence of the seasonal rainfall distribution (Fig. 3).

The depth to the water table ranges from less than $1 \mathrm{~m}$ (rainy season) up to 15 m (dry season), without any spatial relationship within the basin. The potentiometric levels are always higher than the river bed; this observation is also valid for the more densely monitored Ara subcatchment (Fig. 1), where seasonal perched ground water was recognized. The water table peak is reached in August, and the drop begins simultaneously with the rainfall (Fig. 3C). The water table fluctuations range between 3.3 and $12.5 \mathrm{~m}$ in 2003 and between 2.4 and $10.0 \mathrm{~m}$ in 2004, and appears synchronous at the catchment scale.

\subsection{Hydrochemistry}

Sampled waters are slightly acidic $(\mathrm{pH}=5-7)$, present a low mineralization (<400 $\mu$ S. $\mathrm{cm}^{-1}$; Fig. 4), and are of $\mathrm{HCO}_{3}-\mathrm{Ca}, \mathrm{Mg}$ type (some groundwaters enriched in $\mathrm{Cl}$ and $\mathrm{NO}_{3}$ near villages were considered polluted). The lowest EC is observed in rainfall waters $\left(10 \pm 2 \mu \mathrm{S} . \mathrm{cm}^{-1}\right)$. Except for the very first flood events that may flush the salt accumulated for the dry season, EC measured in river waters is low $(<70$ $\mu \mathrm{S} . \mathrm{cm}^{-1}$ ). The EC displays typical changes during flood events, with low values at the peak flow followed by a recovery of the initial value (Fig. 5A). The phreatic 
groundwater EC is of $155 \pm 70 \mu \mathrm{S} . \mathrm{cm}^{-1}$ but EC is higher for the deeper fractured bedrock $\left(288 \pm 80 \mu \mathrm{S} . \mathrm{cm}^{-1}\right)$. Perched groundwaters show lower EC, within the range of 30 to $130 \mu \mathrm{S} . \mathrm{cm}^{-1}$ (Fig. 4).

Ionic ratios show little changes through time and, for river waters, elements related to chemical weathering $\left(\mathrm{Si}, \mathrm{Ca}, \mathrm{Mg}, \mathrm{Na}\right.$, and $\mathrm{HCO}_{3}$ ) vary proportionally to $\mathrm{EC}$ (Fig. 5A); potassium is an exception, probably because of its sensitivity to biochemical cycles [4]. Changes in ionic fluxes appear to mimic the peak flow with a time-lag of a few hours (Fig. 5B), a possible consequence of subcatchments with different weathering rates.

\section{Discussion}

\subsection{Hydrological processes}

Hydrodynamics, hydrochemical and geophysical data were crossed to decipher the natural processes in play in the basin (Fig. 6).

For groundwater flow, the water table is always in a higher position than the drainage network. The weathered bedrock acts as a storage reservoir, with a weak transmissivity (estimated of the order of $10^{-4} \mathrm{~m}^{2} \mathrm{~s}^{-1},[16]$ ). During the rainy season, a water table rise is observed in each point, thus indicating that recharge occurs mainly by vertical infiltration of rainfall.

The seasonal discharge of poorly mineralized river waters suggests that most of the river water originates from the sub-surface. Observations of springs on hillslopes and the occurence of perched groundwaters both suggest important subsurface flow. The river discharge may therefore be considered as a mixing of surface runoff (Hortonian runoff and flow over saturated soils) with subsurface flow from the upper metres of the soil (Fig. 6). The aquifer discharge may occur mainly through evapotranspiration processes, although locally, groundwater discharge could also occur through some deep fractures of higher porosity [1].

\subsection{Water balance}

Two methods were applied to constrain the hydrological balance. The first one considers natural water table fluctuation induced from rainfall events over a short period [7]: $R=h \times n$, where $R$ represents recharge $\left(\mathrm{L} \mathrm{T}^{-1}\right), h$ the water table rise $\left(\mathrm{L} \mathrm{T}^{-}\right.$ ${ }^{1}$ ) and $n$ the specific yield (\%). For our study case, $h$ is the average seasonal rise of 
the water table (Fig. 3C). The calculated recharge for a $n$ value therefore represents a minimum estimate, considering that discharge may occur during the rainy season. A range of values of 1 to $5 \%[9,13]$ was considered to quantify the influence of $n$ on $R$ (Table 1). The estimated recharge ranges between 5 to $24 \%$ of the total rainfall amount, in agreement with other regional estimates [13]. These recharge rates are compatible with discharge by evapotranspiration during the dry season. At slope scale, an estimate of groundwater discharge using Darcy's law (T of $10^{-4} \mathrm{~m}^{2} \mathrm{~s}^{-1}$, piezometric gradients of 2-3\%) shows that ETP rates of about $15-20 \mathrm{~mm} \mathrm{day}^{-1}$ for forest galleries would be necessary to prevent any inflow to the river (Fig. 6). Although this range appears to be higher than the computed ETP (5-6 mm day ${ }^{-1}$ ), clothesline and oasis effects are known to increase ETP rates in forest gallery, possibly at a factor higher than 2 [10]; further research would however be needed to better constrain surface water - groundwater relationships.

The second approach, based on hydrograph decomposition [2], was used to discriminate direct runoff and subsurface flow in river discharge. A two-component, one-tracer (EC) hydrograph separation model was considered (EC being representative of the ionic load: e.g., $R^{2}$ of 0.91 between $E C$ and $\mathrm{Ca}$, Fig. 5A). The different signatures were assumed to be roughly constant in time and space; computations were done for the $\mathrm{K}$ station (Fig. 1). The end-members values (direct

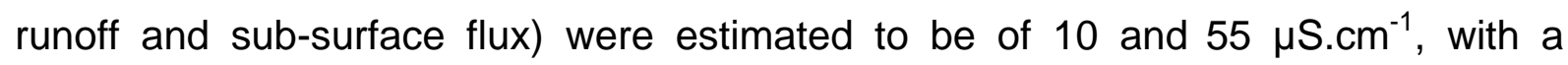
standard-deviation of 2 and 6, respectively. A Monte Carlo approach was used to estimate the uncertainty; sub-surface flux makes up to $68 \pm 13$ and $83 \pm 13 \%$ of the streamflow in 2003 and 2004, respectively. This suggests that the river flow deficit has not a single origin, but results in a decrease in both surface and subsurface flow. This conclusion differs from those of other studies performed in the Sahel, where Hortonian runoff was shown to have increased following soil crusting (e.g., [26]).

\section{Conclusion}

In central tropical Benin, at the $586 \mathrm{~km}^{2}$ Donga basin's scale, runoff and subsurface flow are the main contributors to streamflow, with little or no input from the aquifer. This result supplements previous studies performed in smaller $\left(30 \mathrm{~km}^{2}\right)$ neighbouring catchments [14] where land clearance was shown to have increased surface runoff, in accordance with the global trend [25]. These results will first help in 
improving the relevancy of the regional hydrological models $[3,20,27]$; on a broader perspective, they will also contribute to a better understanding of the origin of the decrease in river discharge observed for the past decades in West Africa $[18,23]$.

Acknowledgments An important part of the field data were collected by S. Afouda, M. Arjounin, J.M. Bouchez, F. Jacquin, F. Malinur and T. Ouani (IRD, Benin). The rainfall and surface water data were criticized by $L$. Le Barbé and C. Peugeot (IRD). The Direction of Hydraulics of Djougou (Benin) allowed access to the wells and to their technical archives. The villagers of the study area are thanked for their hospitality and for their assistance for some surveys. This work was made possible by a PhD grant from Côte d'Ivoire (B. Kamagaté) and by the french national programs ECCO-PNRH (2003-2006) and ORE (2002-2010). Support by the european project AMMA-EU (2005-2009) is also acknowledged. 


\section{Introduction}

En Afrique de l'ouest sous régime de mousson, la sécheresse en cours depuis le début des années 1970, caractérisée par une diminution de l'ordre de $20 \%$ à 30\% de la pluviométrie $[18,21]$ a engendré une diminution encore plus importante des débits [23]. Au centre du Bénin, sur la zone d'étude considérée, le déficit des écoulements, de l'ordre de 40\%, correspond à une diminution de la pluviométrie de 15 à 20\% [18]. Cette relation implicitement non linéaire entre pluie et débit, ainsi que sa variabilité spatiotemporelle, sont encore mal comprises à l'échelle régionale ; les principales études ont jusqu'à présent été menées à partir de données hydrodynamiques acquises sur de grands bassins versants (de l'ordre de 10000 à $100000 \mathrm{~km}^{2}$ ), par des approches indirecte ou conceptuelle (tests statistiques; calculs de coefficients de tarissement [23, 24]). L'explication proposée est que la diminution relativement plus importante des débits trouve son origine dans une réduction durable des apports en eau souterraine. Si la validation de cette hypothèse nécessite d'être effectuée par une analyse des processus physiques à une échelle comparable à celle des observations de surface (données piézométriques disponibles sur plusieurs milliers de $\mathrm{km}^{2}$, sur la période pluridécennale des observations de débits), des éléments de réponse peuvent cependant être apportés par la caractérisation des processus physiques affectant les différents réservoirs hydriques d'un bassin versant représentatif, étudié sur des années à pluviométrie et écoulements contrastés. En Afrique subsaharienne, les rares études existantes à fine échelle (bassins versants expérimentaux de l'ordre du km², [e.g., 6]) montrent que la discrimination des écoulements est améliorée par une approche pluridisciplinaire des différents termes du bilan.

Le programme international AMMA (Analyse Multidisciplinaire de la Mousson Africaine; http://amma-international.org) a été initié en vue d'améliorer les connaissances sur la mousson ouest-africaine et de son impact hydrologique. Différents sites instrumentés font l'objet, depuis 2003, de suivis renforcés, dont celui du bassin versant de la Donga (Ouémé supérieur) au Bénin (Fig. 1). Sa superficie $\left(586 \mathrm{~km}^{2}\right.$ ) en fait un objet hydrologique intermédiaire entre les grands bassins régionaux et les bassins expérimentaux; cette échelle rend possible une caractérisation des processus hydrologiques tout en permettant, en terme de bilan, 
une intégration suffisamment représentative des variations spatiotemporelles naturelles. A l'échelle régionale, de même que l'analyse de la variation spatiotemporelle de la pluviométrie [19] ou de la caractérisation spatiale des sols [14], la connaissance des processus surface-souterrain est un élément fondamental pour appréhender la variabilité temporelle des ressources hydriques.

Dans cette note, nous présentons les premiers résultats des suivis hydrodynamiques (piézométrie et débits) et hydrochimiques (physico-chimie et ions majeurs) réalisés sur le bassin versant de la Donga au cours de deux années à pluviométrie contrastée (1514 mm en 2003, $1140 \mathrm{~mm}$ en 2004). L'analyse des processus en jeu sur le bassin, appuyée par des mesures de géophysique de subsurface (résistivité), permet d'obtenir une première estimation du bilan hydrologique. Le schéma des flux hydriques proposé relativise, à l'échelle de l'étude, l'impact de l'aquifère sur les flux de surface et suggère une importance prépondérante des écoulements de surface et de subsurface comme facteurs de la variabilité interannuelle des débits observés à l'exutoire.

\section{Zone d'étude}

Le bassin de la rivière Donga est un affluent de l'Ouémé, le plus grand fleuve du Bénin (523 km) dont la haute vallée couvre une superficie de $14300 \mathrm{~km}^{2}$ [17]. ॥ se situe au centre-ouest du Bénin (Fig. 1), sous climat tropical de type soudanoguinéen à saisons contrastées (alternance d'une saison sèche et d'une saison pluvieuse). Sur ce bassin versant, la pluviométrie moyenne annuelle observée à la station météorologique de Djougou, sur la période 1950-2004, est de 1195 mm (1280 mm de 1950 à 1969, 1150 mm de 1970 à 2004) ; l'essentiel des pluies (60 \%) se concentre entre juillet et septembre. L'évapotranspiration potentielle (ETP) calculée par la méthode de Penman-Monteith [10] est de $1390 \mathrm{~mm} \mathrm{an}^{-1}$ (2002-2005); à l'échelle mensuelle, elle montre une faible variabilité saisonnière, avec un maximum de 5 à $6 \mathrm{~mm}_{\text {jour }}{ }^{-1}$ en saison sèche et un minimum de 3 à $4 \mathrm{~mm}$ jour $^{-1}$ en saison des pluies.

La topographie du bassin est vallonnée, avec des altitudes comprises entre $520 \mathrm{~m}$ à l'amont (ouest) et $340 \mathrm{~m}$ à l'exutoire (est) avec une pente moyenne de la 
rivière de $1,7 \mathrm{~m} \mathrm{~km} \mathrm{~km}^{-1}$. Les roches sont essentiellement des formations métamorphiques de la bordure est du craton ouest-africain, datées du Protérozoïque supérieur et constituées de granites, de granito-gneiss et de schistes [1, 5]. Les altérites, principalement silteuses et argileuses sur une épaisseur de 10 à $25 \mathrm{~m}$, constituent l'aquifère de la nappe libre. Localement et plus en profondeur, des fractures du socle peuvent constituer un réservoir pérenne, en continuité hydraulique avec la couverture altérée du socle [16].

Les sols régionaux, de type "ferrugineux tropicaux lessivés" [12] sont de nature variable en fonction de leur position topographique, de la nature des roches sous-jacentes ou de l'anthropisation du site (sols localement hydromorphes, indurés, peu lessivés ou lessivés [11]). La végétation climacique est constituée d'une savane à Isoberlinia sp. à couvert herbacé continu (Andropogon sp., Hypparhenia sp.). Cette végétation naturelle est aujourd'hui largement dégradée en une mosaïque de champs (igname, manioc, sorgho, maïs, coton). A l'aval des versants, les principaux axes de drainage sont bordés sur 10 à 20 m par une dense forêt-galerie.

L'activité socio-économique, hormis la ville de Djougou (20 000 habitants) est essentiellement rurale et basée sur des cultures pluviales (absence d'irrigation). La densité de la population, proche de 30 hab. $\mathrm{km}^{-2}$, connaît une croissance annuelle de $4,9 \% \mathrm{an}^{-1}$ (1992-2002) ; l'essentiel des ressources en eau à usage domestique provient d'une multitude de puits villageois disséminés sur l'ensemble du bassin. Des suivis de terrain des puisages quotidiens, effectués en 2003 dans 6 villages représentatifs, indiquent une consommation domestique de l'ordre de $21 \mathrm{~L}$ hab. jour' ${ }^{1}$, proche de celle de $17 \mathrm{~L}$ hab. jour ${ }^{-1}$ estimée pour l'ensemble du haut-Ouémé [15]. Compte tenu de la population du bassin de la Donga, cela implique des prélèvements anthropiques faibles, de l'ordre de $0,2 \mathrm{~mm} \mathrm{an}^{-1}$.

\section{Données}

Des données préliminaires sur l'organisation de la couverture d'altération ont été obtenues en mai 2003 par des prospections géophysiques basées sur la résistivité électrique [8]. Dans ce type de milieu, ce paramètre présente des contrastes importants en fonction de la nature des roches, des teneurs en eau ou en 
argile. La méthode de la tomographie électrique [22] a été mise en œuvre sur plusieurs versants du bassin à une échelle pluri-hectométrique. En parallèle aux mesures, des relevés géologiques ont été effectués pour contraindre l'interprétation.

Les données hydrodynamiques sont issues de 16 pluviomètres, de 26 puits suivis en piézométrie et de 3 stations limnimétriques ; ces stations représentent, d'amont en aval, des basins emboîtés de superficie croissante (Ara- pont (A), 13

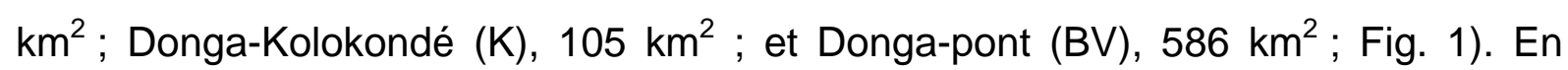
complément, à plus fine échelle, des données issues d'un site à l'amont du bassin où 9 piézomètres ont été crépinés à différentes profondeurs $(2,10$ et $20 \mathrm{~m})$ sont disponibles depuis 2004 (Fig. 1). Les pluviomètres enregistreurs permettent de discriminer les événements par cumuls de 0,5 $\mathrm{mm}$. Les niveaux de la nappe libre ont été enregistrés à pas de temps de 30 min (13 sites) et/ou par lectures tri-journalières (23 sites). Les trois stations limnimétriques considérées, étalonnées, ont bénéficié d'enregistrements à pas de temps de 5 min pour les deux stations à l'amont $(A, K)$ et de 30 min pour la station à l'aval (exutoire). L'ensemble des piézomètres et des échelles limnimétriques ont été nivelés par GPS différentiel pour caractériser la distribution spatiale des potentiels hydrauliques [16].

Les données hydrochimiques sont constituées de suivis mensuels à trimestriels des paramètres physico-chimiques (température, $\mathrm{pH}$, conductivité électrique (CE à $\left.25^{\circ} \mathrm{C}\right)$ ) sur une vingtaine de puits et forages. Des mesures ont également été effectuées sur le cumul d'une dizaine d'événements pluvieux sur 5 pluviomètres de l'amont du bassin, ainsi que dans les eaux de surface, en divers points du bassin (sources, affluents et cours principal de la Donga). La station limnimétrique de Donga-Kolokondé (K, Fig. 1) fait l'objet depuis juin 2003 d'un suivi automatique de la $C E$ à pas de temps de $30 \mathrm{~min}$; sur les piézomètres du site expérimental de l'amont du bassin, des enregistrements de la CE à pas de temps de $30 \mathrm{mn}$ sont également effectués depuis avril 2004. En parallèle à ces mesures, des analyses de la chimie des ions majeurs $\left(\mathrm{Ca}^{2+}, \mathrm{Mg}^{2+}, \mathrm{Na}^{+}, \mathrm{K}^{+}\right.$pour les cations, $\mathrm{HCO}_{3}{ }^{-}$, $\mathrm{SO}_{4}{ }^{2-}, \mathrm{NO}_{3}{ }^{-}, \mathrm{Cl}^{-}$pour les anions) ont été réalisés sur des eaux de pluie (18 analyses), de la nappe (23 puits et 15 forages), de sources temporaires (8 analyses) et du cours principal de la Donga (une douzaine d'analyses par station limnimétrique). Pour les eaux de la Donga, un échantillonnage à haute fréquence a également pu être 
effectué sur 3 crues majeures en 2003. L'ensemble des échantillons, filtrés in-situ, a été analysé avec une précision de $5 \%$ par électrophorèse capillaire (cations et $\mathrm{SO}_{4}{ }^{2-}$, $\left.\mathrm{NO}_{3}{ }^{-}, \mathrm{Cl}^{-}\right)$ou titration acide $\left(\mathrm{HCO}_{3}^{-}\right)$au laboratoire HydroSciences de Montpellier (France).

\section{Résultats}

\subsection{Géophysique de sub-surface}

Plusieurs profils de résistivité électrique transversaux aux talwegs ont été réalisés par tomographie en différents points du bassin $[8,16]$. Un exemple typique de profil de résistivité obtenu en amont du bassin est présenté Figure 2 ; ce profil montre une hétérogénéité importante de l'altération à une échelle hectométrique. Les formations argileuses et/ou argilo-sableuses électriquement conductrices sont organisées en lentilles parfois affleurantes; cette distribution suggère que des nappes perchées peuvent se mettre en place, les formations argileuses constituant des niveaux imperméables. Des cuirasses superficielles se notent par de fortes résistivités électriques. Le socle, électriquement résistant, se rencontre entre 15 et $25 \mathrm{~m}$ sous la surface. Son modelé présente de faibles ondulations sans relation avec la topographie. Localement, des formations massives de plus forte résistance (e.g., quartzites), propices au ruissellement, affleurent (Fig. 2).

\subsection{Hydrodynamique}

Pour les deux années suivies, les pluies présentent une saisonnalité similaire avec un pic mensuel en juillet - août (Fig. 3A). La pluviométrie présente une diminution du cumul de 25\% (1514 mm en 2003, 1140 mm en 2004, Tableau 1). Cette différence se trouve accentuée (64\%) dans les écoulements de surface à l'exutoire (429 mm en 2003, 156 mm en 2004), avec des coefficients d'écoulement à l'exutoire respectivement de 28,3 et 13,7\% (Tableau 1). Les mêmes conclusions peuvent être apportées pour les sous-bassins emboîtés A et K (Tableau 1). En termes de processus, l'écoulement présente une dynamique rapide, avec des crues journalières pouvant atteindre $140 \mathrm{~m}^{3} \mathrm{~s}^{-1}$ (Fig. 3B). A l'échelle saisonnière, la durée de l'écoulement apparaît plus courte en 2004 qu'en 2003 (120 contre 210 jours), tributaire de l'occurrence de pluies rapprochées. 
La profondeur de la nappe est comprise entre $15 \mathrm{~m}$ (saison sèche) et moins de $1 \mathrm{~m}$ sous le sol (saison des pluies), en relative indépendance de la position dans le bassin. A cette échelle, la piézométrie de la nappe est toujours en position haute par rapport au lit de la rivière. Cette observation est également valable à l'échelle du sous-bassin versant d'Ara, plus densément équipé. Sur ce site, les piézomètres crépinés à 10 et $20 \mathrm{~m}$ montrent une piézométrie identique (nappe phréatique pérenne des altérites) tandis que ceux crépinés à $2 \mathrm{~m}$ captent un niveau saturé à potentiel plus élevé (nappe perchée temporaire). Le maximum piézométrique de la nappe phréatique est atteint en août-septembre, après une hausse plurimétrique des niveaux, sans répartition particulière des amplitudes de fluctuation sur le bassin. De même que les écoulements de surface, la décrue piézométrique s'amorce dès la fin des précipitations régulières pour atteindre un minimum en mars-avril (Fig. 3C). Ces fluctuations piézométriques montrent, en comparaison interannuelle, une différence moindre que celle des débits (Fig. 3B): l'amplitude des chroniques varie de 3,3 à 12,5 m (moyenne : 7,4 $\pm 2,8 \mathrm{~m}$ ) en 2003 et de 2,4 à 10,0 m (moyenne : 5,3 \pm 2,3 m) en 2004. La nappe phréatique des altérites apparaît donc de dynamique synchrone à l'échelle du bassin, avec une continuité hydraulique effective à l'échelle des versants.

\subsection{Hydrochimie}

Les eaux échantillonnées sont peu minéralisées (hormis quelques sites pollués enrichis en $\mathrm{NO}_{3}$ et $\mathrm{Cl}$ ) avec des $\mathrm{CE}$ généralement inférieures à $400 \mu \mathrm{S} \mathrm{cm}^{-}$ ${ }^{1}$ (Fig. 4). Les eaux sont à tendance acide ( $\mathrm{pH}=5$ à 7 ) et de faciès chimique majoritairement bicarbonaté calco-magnésien, en accord avec la nature silicatée des roches. La température des eaux de la nappe $\left(27\right.$ à $\left.28^{\circ} \mathrm{C}\right)$ est légèrement supérieure à la température moyenne interannuelle à la station de Djougou $\left(26,4{ }^{\circ} \mathrm{C}\right)$.

Les pluies montrent des CE faibles, de l'ordre de $10 \mu \mathrm{S} \mathrm{cm}^{-1}$. Ces valeurs sont logiquement inférieures à celles des eaux de surface (Donga et affluents) dont la $\mathrm{CE}$, hormis les tous premiers événements lessivants de la saison, reste faible en valeur absolue $\left(<70 \mu \mathrm{S} \mathrm{cm}^{-1}\right)$. En valeur relative, la CE montre des fluctuations systématiques en cours de crue : à une chute de CE en début d'hydrogramme, succède un recouvrement lent (quelques jours) de la $C E$ initiale (Figure 5A, qui représente une crue composite à l'exutoire). En termes de flux ioniques, la 
dynamique géochimique montre des variations similaires mais retardées de quelques heures par rapport au débit (Fig. 5B) ; cette diachronie peut s'expliquer par des contributions variables de sous-bassins aux taux d'érosion chimique différents. D'amont en aval du bassin, en période d'inter-crue, une faible augmentation de la CE est perceptible (de $39 \pm 5$ à $65 \pm 7 \mu \mathrm{S} \mathrm{cm}^{-1}$ pour A et BV) ; l'explication la plus probable réside dans une augmentation de la longueur moyenne des versants et donc du temps de transit de l'écoulement de subsurface. A l'échelle saisonnière comme à l'interannuel pour les deux années suivies, aucune tendance à l'augmentation de la CE des eaux de la rivière n'est observée (Fig. 4).

La nappe phréatique (altérites) montre des valeurs nettement supérieures, en moyenne de $155 \pm 70 \mu \mathrm{S} \mathrm{cm}^{-1}$; plus en profondeur, les eaux des forages (socle) montrent des valeurs encore plus élevées $\left(288 \pm 80 \mu \mathrm{S} \mathrm{cm}^{-1}\right)$; ceci témoigne d'un temps de résidence probablement plus important. Sur le site de plus fine investigation à l'amont du bassin, les suivis hydrochimiques effectués en 2004 permettent de préciser ces observations. La nappe perchée $(2 \mathrm{~m})$ montre des CE fluctuant entre 30 et $130 \mu \mathrm{S} \mathrm{cm}^{-1}$ (moyenne de $75 \pm 30 \mu \mathrm{S} \mathrm{cm}^{-1}$ ) alors que celles relatives aux piézomètres crépinés à 10 et $20 \mathrm{~m}$ sont respectivement de l'ordre de 200 et $250 \mu \mathrm{S} \mathrm{cm}^{-1}$ (Fig. 4).

En terme de minéralisation, les rapports ioniques montrent une grande constance dans le temps et l'espace. Pour les eaux de la rivière (Fig. 5A), les éléments majeurs liés à l'altération $\left(\mathrm{Si}, \mathrm{Ca}, \mathrm{Mg}, \mathrm{Na}\right.$ et $\left.\mathrm{HCO}_{3}\right)$ fluctuent proportionnellement à la $\mathrm{CE}$; l'ion $\mathrm{K}$ fait exception et montre un comportement variable, vraisemblablement en raison d'une sensibilité plus grande aux cycles biogéochimiques [4]. Au final, les eaux issues de différentes profondeurs (subsurface, nappe des altérites et socle profond) montrent des rapports ioniques peu différenciés, reflet de l'homogénéité d'ensemble des faciès chimiques des eaux du bassin. 


\section{Discussion}

\subsection{Processus hydrologiques}

Le croisement d'informations de la géophysique, de l'hydrodynamique et de la géochimie permet de reconstituer un schéma cohérent des processus en jeu sur le bassin (Fig. 6).

La nappe phréatique, libre, est sur tout le bassin en position haute par rapport au réseau hydrographique. Elle occupe les versants en continuité hydraulique, comme en témoignent les mesures piézométriques. Les coupes géophysiques montrent qu'elle baigne différents terrains organisés en lentilles silto-argileuses ou sableuses. Au cours de la saison des pluies, elle montre en tout point des fluctuations saisonnières, d'amplitude variable et sans organisation spatiale particulière; la recharge apparaît donc principalement de type direct, par percolation des eaux de pluie et infiltration à travers la zone non-saturée ; en certains points du bassin cependant, l'existence de sols indurés (cuirasse) et/ou d'affleurements du socle peut induire un ruissellement localisé avant infiltration. La nature silto-argileuse à sablo-argileuse du manteau altéritique milite pour un rôle essentiellement capacitif du réservoir ; cette interprétation a été confirmée par essais de pompage, avec des débits spécifiques de l'ordre de $1 \mathrm{~m}^{3} \mathrm{~h}^{-1} \mathrm{~m}^{-1}$ et des perméabilités de l'ordre de $10^{-5} \mathrm{~m}$ $\mathrm{s}^{-1}[16]$.

Le caractère temporaire des eaux de la Donga (absence de "débit de base") traduit une origine superficielle des écoulements. Lors des pics de crue, les eaux présentent une minéralisation très faible, proche de celle des pluies; en période d'inter-crue, les écoulements sont de faible minéralisation, de chimisme voisin de celui des nappes perchées saisonnières tel qu'observé à leurs émergences en de multiples points du bassin [16]. L'écoulement à l'exutoire peut donc être considéré comme composé d'un flux rapide (ruissellement Hortonien sur les surfaces indurées et écoulement sur surfaces saturées) et d'un flux retardé de sub-surface (vidange des nappes perchées), sans contribution significative de la nappe des altérites à l'écoulement. La vidange de la nappe semble donc s'effectuer essentiellement par des transferts verticaux par évapotranspiration (strate arborée) et éventuellement, 
vers des fractures du socle plus en profondeur, via des brèches tectoniques à forte porosité [1] ; cependant, à une échelle de $10000 \mathrm{~km}^{2}$, le haut-Ouémé présente un tarissement annuel, ce qui milite plutôt pour des circulations dans le socle faibles ou d'extension limitée.

\subsection{Bilan hydrologique}

Deux méthodes sont appliquées pour contraindre le bilan hydrologique du bassin. La première est basée sur les fluctuations piézométriques de la nappe libre et a pour objet une estimation de la recharge annuelle. La deuxième est basée sur une décomposition des hydrogrammes de crue et a pour objet de discriminer la part des écoulements rapides et de subsurface dans les flux à l'exutoire.

L'estimation de la recharge par la méthode des fluctuations piézométriques suppose une nappe libre, rechargée par les précipitations en un temps court [7]; elle peut s'exprimer selon l'équation $R=h \times n$, où $R$ représente la recharge $\left(\mathrm{L} \mathrm{T}^{-1}\right), h$ l'amplitude piézométrique observée sur la période considérée $\left(\mathrm{L} \mathrm{T}^{-1}\right)$ et $n$ la porosité efficace (\%, adimensionnel). Pour notre cas d'étude où la recharge s'effectue en quelques mois, l'amplitude piézométrique considérée est la valeur moyenne entre le minimum de saison sèche et le maximum de la saison des pluies (Fig. 3C). L'estimation obtenue pour une valeur $n$ considérée représente alors, du fait (1) d'une probable vidange concomitante à la recharge et (2) d'une amplitude de fluctuation parfois limitée par le niveau du sol, une estimation a minima de la recharge effective. Les prélèvements villageois, susceptibles d'induire un rabattement local de la nappe, ont probablement une influence minime, les fluctuations mesurées dans les piézomètres expérimentaux présentant des amplitudes similaires à celles obtenues dans les puits. L'influence des flux souterrains provenant de l'amont des points de mesure est considérée comme négligeable, les observations réalisées sur des transects piézométriques le long de versants montrant de faibles différences de fluctuation saisonnière (<15\%). Pour quantifier l'impact du choix de $n$ sur la recharge estimée, une gamme de 1 à $5 \%$ a été retenue pour la porosité efficace (Tableau 1), en accord avec les valeurs généralement admises pour les altérites de socle en Afrique de l'Ouest $[9,13]$. La recharge déduite est de 74 à $370 \mathrm{~mm} \mathrm{an}^{-1}$ (2003) et de 53 à $265 \mathrm{~mm} \mathrm{an}^{-1}$ (2004), soit de 5 à $24 \%$ du total pluviométrique. Ces coefficients de recharge sont compatibles avec la gamme de 20 à $30 \%$ obtenue pour des 
aquifères en zone de socle et sous climat soudanien [13]. A l'échelle du bassin, ces chiffres apparaissent compatibles avec des flux d'exfiltration par évapotranspiration pendant les 5 à 6 mois de la saison sèche. A l'échelle des versants (Figure 6), une estimation des flux souterrains peut être effectuée par l'application de la loi de Darcy : sur la base des paramètres de l'aquifère (perméabilité de $10^{-5} \mathrm{~m} / \mathrm{s}$, épaisseur saturée de 10 à $20 \mathrm{~m}$, gradients hydrauliques de l'ordre de 2 à 3\%), les flux parvenant aux talwegs sont de l'ordre de $0,3 \mathrm{~m}^{3} \mathrm{~m}^{-1}$ jour $^{-1}$. En considérant une largeur de forêts-galeries de $20 \mathrm{~m}$, un flux d'évapotranspiration de l'ordre de 15 à 20 $\mathrm{mm} \mathrm{jour}^{-1}$ serait alors suffisant pour empêcher toute contribution du souterrain au réseau hydrographique. Selon [10], en raison de l'effet d'oasis et de leur organisation linéaire, I'ETP des forêts galeries peut être d'au moins un facteur 2 supérieure à l'ETP de 5 à $6 \mathrm{~mm}$ jour $^{-1}$ en saison sèche, calculée selon les seuls paramètres atmosphériques. En première approche, l'ordre de grandeur des flux souterrains apparaît donc compatible avec des flux d'exfiltration gouvernés par évapotranspiration; des études complémentaires seraient cependant nécessaires pour mieux quantifier les flux hydriques à l'aval des versants.

En accord avec les processus identifiés, une décomposition de l'hydrogramme en deux écoulements (ruissellement, RR, et flux de subsurface, SS) a été retenue. Les hypothèses classiques [2] suivantes ont été adoptées: (1) invariance spatiotemporelle de la signature des réservoirs contributeurs et (2) la CE représente une valeur représentative de la charge ionique issue de l'altération. Pour notre cas d'étude, des variations temporelles du chimisme sont cependant notées en subsurface (Fig. 4) et $\mathrm{K}^{+}$évolue indépendamment de la CE (Fig. 5A); ces écarts restent suffisamment mineurs (e.g., $R^{2}$ de 0,91 entre $C E$ et $C a$, Figure $5 A$ ) pour justifier en première approche les hypothèses. Des observations réalisées à la station " $\mathrm{A}$ " montrent des CE proches de celles de l'eau de pluie durant les pics de crue $(8<\mathrm{CE}$ $\left.<17 \mu \mathrm{S} \mathrm{cm}^{-1}\right)$, ce qui peut justifier de l'assimilation de la CE des pluies $(10 \pm 2 \mu \mathrm{S} \mathrm{cm}$ $\left.{ }^{1}\right)$ au pôle RR. En considérant les valeurs de CE mesurées aux 3 stations en période d'inter-crue, une valeur de $55 \pm 6 \mu \mathrm{S} \mathrm{cm}^{-1}$ a été fixée au pôle SS ; cette valeur est dans la gamme de celles observées pour les nappes perchées $\left(75 \pm 30 \mu \mathrm{S} \mathrm{cm}^{-1}\right)$. Les calculs se rapportent à la station K (Fig. 1), équipée d'un enregistreur de CE sur les deux années considérées, aux valeurs de CE proches de celles de l'exutoire $(53 \pm 8$ et $65 \pm 7 \mu \mathrm{S} \mathrm{cm}^{-1}$ respectivement, en période d'inter-crue). Pour tenir compte de 
l'incertitude sur les pôles, une simulation a été réalisée par la méthode de Monte Carlo (tirage des valeurs des pôles dans des lois normales de moyenne et écart-type précédemment donnés). Les flux de subsurface représentent $68 \pm 13 \%$ et $83 \pm 13 \%$ de l'écoulement total, respectivement en 2003 et 2004 ; à l'échelle intra-saisonnière, aucune évolution significative des contributions n'est observée. Sur cette partie du bassin versant, les sommes des écoulements rapides et de sub-surface peuvent donc être estimée respectivement à 140 et 297 mm en 2003, et à 18 et 88 mm en 2004. Pour ces deux années à écoulement contrasté, ces calculs suggèrent que la baisse des écoulements n'a pas une origine unique, mais résulte d'un déficit combiné des flux de surface et de subsurface. Ce processus s'avère donc distinct de celui observé plus au nord au Sahel, où le déficit pluviométrique combiné au déboisement a provoqué la seule augmentation du ruissellement Hortonien sur surfaces encroûtées (e.g., [26]); en zone soudanienne, un sol plus développé favorise l'infiltration locale et les flux de subsurface, dont la réponse au déficit pluviométrique peut être différente en amplitude de celle du ruissellement.

\section{Conclusion}

Cette étude, basée sur une approche couplée hydrodynamique et hydrochimique des flux hydriques, montre que l'origine des écoulements sur le bassin versant de la Donga est à dominante superficielle. Ce résultat précise et complète, pour le haut Ouémé, des études antérieures sur un sous-bassin moins étendu $\left(30 \mathrm{~km}^{2}\right)$ où l'impact de la mise en culture a engendré une augmentation du ruissellement et une réduction de l'évapotranspiration [14], en accord avec la tendance observée à l'échelle globale [25]. A terme, par une meilleure prise en compte des processus hydrologiques et de la variabilité interannuelle des flux, une amélioration des modèles hydrologiques établis pour le Haut Ouémé [3, 20, 27] est attendue ; en retour, ces modèles permettront de valider la cohérence des gammes de valeurs estimées pour les différents paramètres du bilan hydrologique du bassin.

Ces résultats constituent une première étape dans l'étude hydrologique de ce bassin versant. Dans le cadre du programme AMMA, un suivi à plus long terme permettra de préciser encore davantage l'importance respective du ruissellement et des écoulements de subsurface dans la variabilité interannuelle des débits. Dans 
une perspective plus large, cette étude représente également une contribution à une meilleure connaissance de la variabilité spatio-temporelle des flux surface-souterrain et de son impact sur l'hydrologie de l'Afrique tropicale $[18,23]$.

Remerciements Une part importante de l'acquisition de données de terrain a été assurée par S. Afouda, M. Arjounin, J.M. Bouchez, F. Jacquin, F. Malinur et T. Ouani, techniciens de l'IRD au Bénin. Les traitements des données pluviométriques et hydrométriques ont été coordonnés respectivement par L. Le Barbé et $\mathrm{C}$. Peugeot (IRD). La Direction de l'Hydraulique de Djougou (MMEH, Bénin) a permis l'accès aux données et aux infrastructures hydrauliques du bassin. Les villageois de la zone étudiée sont remerciés pour leur accueil et leur participation active à certains suivis. Ce travail a été rendu possible par une bourse de thèse de la Côte d'Ivoire (B. Kamagaté), par les financements institutionnels des programmes français ECCOPNRH (2003-2006) et ORE (2002-2010), ainsi que par le soutien du projet européen AMMA-EU (2005-2009).

\section{Références}

[1] P. Affaton, Le Bassin des Voltas (Afrique de l'Ouest) : une marge passive, d'âge protérozoïque supérieur, tectonisée au panafricain (600 $\pm 50 \mathrm{Ma}$ ), Thèse Géologie, Université d'Aix-Marseille 3, 1987. ORSTOM Editions, collection Etudes et Thèses, Paris, France, 1990, 499 p.

[2] J.L. Boeglin, Y. Tardy, Erosion chimique et mécanique sur le bassin amont du Niger (Guinée, Mali). Découpage en quatre écoulements, C. R. Acad. Sci. Paris lla 325 (1997) 125-131.

[3] H. Bormann, T. Fass, S. Giertz, B. Junge, B. Diekkrüger, B. Reichert, A. Skowronek, From local hydrological process analysis to regional hydrological model application in Benin: Concept, results and perspectives, Phys. Chem. Earth 30 (2005) 347-356. 
[4] J.J. Braun, B. Dupré, J. Viers, J.R. Ndam Ngoupayou, J.P. Bedimo Bedimo, L. Sigha-Nkamdjou, R. Freydier, H. Robain, B. Nyeck, J. Bodin, P. Oliva, J.L. Boeglin, S. Stemmler, J. Berthelin, Biogeohydrodynamic in the forested humid tropical environment: the case study of the Nsimi small experimental watershed (south Cameroon), Bull. Soc. Geol. Fr. 173 (2002) 347-357.

[5] BRGM/OBM, Carte géologique au 1/200 $000^{\mathrm{e}}$ feuille de Djougou-Parakou-Nikki, Office Béninois des Mines, Cotonou, République du Bénin, 1984.

[6] P. Chevallier, O. Planchon, Hydrological processes in a small humid savanna basin (Ivory Coast), J. Hydrol. 151 (1993) 173-191.

[7] R.S. Crosbie, P. Binning, J.D. Kalma, A time series approach to inferring groundwater recharge using the water table fluctuation method, Water Resour. Res. 41 (2005) doi: 10.1029/2004WR003077.

[8] M. Descloitres, M. Wubda, Y. Le Troquer, Prospections géophysiques sur le bassin versant d'Ara : électrique 2D et électromagnétisme EM34 ; Compterendu de mission du 5 au 14 mai 2003, Rapport technique IRD, Ouagadougou, Burkina-Faso, 2003, 15 p.

[9] M. Engalenc, Méthode d'étude et de recherche de l'eau souterraine des roches cristallines de l'Afrique de l'ouest, CIEH, Maisons-Alfort, France, 1978, 318 p.

[10] FAO, Crop Evaporation - Guidelines for computing crop water requirements. FAO Irrigation and Drainage paper, 56, Rome, 1998. Available on line at: http://www.fao.org/docrep/X0490E/X0490E00.htm

[11] P. Faure, Carte pédologique de reconnaissance au $1 / 200000^{\mathrm{e}}$, feuille de Djougou, Orstom edition, Paris, France, 1977.

[12] P. Faure, B. Volkoff, Some factors affecting regional differentiation of the soils in the Republic of Benin (West Africa), Catena 32 (1998) 281-306. 
[13] C. Filippi, F. Milville, D. Thiery, Evaluation de la recharge naturelle des aquifères en climat Soudano-Sahélien par modélisation hydrologique globale : application à 10 sites au Burkina-Faso, J. Sci. Hydrol. 35 (1990) 29-48.

[14] S. Giertz, B. Junge, B. Diekkrüger, Assessing the effects of land use change on soil physical properties and hydrological processes in the sub-humid tropical environment of West Africa, Phys. Chem. Earth. 30 (2005) 485-496.

[15] K. Hadjer, T. Klein, M. Schopp, Water consumption embedded in its social context, north-western Benin, Phys. Chem. Earth 30 (2005) 357-364.

[16] B. Kamagaté, Fonctionnement hydrologique et origine des écoulements sur un bassin versant en milieu tropical de socle au Bénin : bassin versant de la Donga (haute vallée de l'Ouémé). Thèse de doctorat, Université de Montpellier II, France, 2006, 319 p.

[17] L. Le Barbé, G. Alé, B. Millet, H. Texier, Y. Borel, R. Gualde, Les ressources en eaux superficielles de la République du Bénin, IRD éditions, Collection Monographies Hydrologiques n¹1, Paris, France, 1993, 469 p.

[18] T. Lebel, T. Vischel, Climat et cycle de l'eau en zone tropicale : un problème d'échelle, C. R. Geoscience 337 (2005) 29-38.

[19] M. Le Lay, S. Galle, Variabilité interannuelle et intra-saisonnière des pluies aux échelles hydrologiques. La mousson ouest-africaine en climat soudanien, J. Sci. Hydrol. 50 (2005) 509-524.

[20] M. Le Lay, S. Galle, How changing rainfall regimes may affect the water balance: a modelling approach in West Africa, In Regional Hydrological Impacts of Climatic Change - Hydroclimatic Variability AISH 296 (2005), pp. 203-210.

[21] Y. L'Hôte, G. Mahé, B. Some, J.P. Triboulet, Analysis of a Sahelian annual rainfall index from 1896 to 2000; the drought continues, J. Sci. Hydrol. 47 (2002) 563-572. 
[22] M. H. Loke, Electrical imaging surveys for environmental and engineering studies. Technical note of Geotomo Software, 2000. Available on line at: http://www.geoelectrical.com

[23] G. Mahé, J.C. Olivry, Evaluation des apports en eau douce à l'Atlantique depuis les côtes de l'Afrique intertropicale (1951-1989), C. R. Acad. Sci. Paris Ila 328 (1999) 621-626.

[24] G. Mahé, J.C. Olivry, R. Dessouassi, D. Orange, F. Bamba, E. Servat, Relation eaux de surface - eaux souterraines d'une rivière tropicale au Mali, C. R. Acad. Sci. Paris Ila 330 (2000) 689-692.

[25] A. Perrier, A. Tuzet, Le cycle de l'eau et les activités au sein de l'espace rural. Enjeux globaux, solutions locales et régionales, C. R. Geoscience 337 (2005) 39-56.

[26] L. Seguis, B. Cappelaere, G. Milesi, C. Peugeot, S. Massuel, G. Favreau, Simulated impacts of climate change and land-clearing on runoff from a small Sahelian catchment, Hydrol. Process. 18 (2004) 3401-3413.

[27] N. Varado, I. Braud, S. Galle, M. Le Lay, L. Seguis, B. Kamagate, C. Depraetere, Multi-criteria assessment of the Representative Elementary Watershed approach on the Donga catchment (Benin) using a downward approach of model complexity, Hydrology and Earth System Sciences 10 (2006) 427-442. 


\section{Légendes}

\section{Figure 1}

- Localisation du bassin versant de la Donga au Bénin (encart, symbole étoilé) et détails du dispositif de suivi hydrologique (2003-2004).

- Location of the Donga watershed in Benin (black star symbol, small inset) and details of the hydrological survey network (2003-2004).

\section{Figure 2}

- Coupe de distribution des résistivités sur un versant représentatif du sous-bassin d'Ara, mai 2003 (localisée à "PG", Fig. 1). L'équivalence entre résistivité et type de terrain est proposée sur la base de mesures de résistivité sur affleurement.

- Resistivity cross section in the Ara watershed in May 2003 (located as "PG" in Fig. 1). The geological interpretation is based on resistivity measurements made on different outcropping surfaces.

\section{Figure 3}

- Lames journalières précipités (A), lames journalières écoulées à l'exutoire du bassin (B) et profondeur moyenne journalière de la nappe (C) pour les années 2003 et 2004.

- Daily rainfall (A), daily runoff at the watershed outlet (B) and mean daily water table depth (C) for the years 2003 and 2004

\section{Figure 4}

- Suivis des fluctuations de la conductivité électrique (CE) des eaux.

- Seasonal EC surveys of surface and ground waters.

\section{Figure 5}

- Fluctuations typiques de la chimie des eaux mesurées à l'exutoire au cours de la crue composite du 01/08/2003. A : concentrations (meq/L), B : Flux (eq/s).

- Typical changes in surface water chemistry during the composite flood of $1^{\text {st }}$ of August, 2003 at the watershed outlet. A: ionic content (meq/L), B: Flux (eq/s). 


\section{Figure 6}

- Schéma conceptuel des flux hydriques à l'échelle d'un versant, d'après les observations hydrologiques, géochimiques et géophysiques réalisées sur le bassin de la Donga. P : Pluie, E : évapotranspiration, If : infiltration, $\mathrm{R}$ : recharge, RR : ruissellement rapide (ruissellement Hortonien et sur surface saturée), SS : flux de subsurface, Fp : flux profond. Les niveaux saisonniers minimum et maximum de la nappe phréatique sont indiqués en tiretés.

- Conceptual cross-section of the water flux at a hillslope scale, according to the hydrological, geochemical and geophysical observations made in the Donga catchment. P: rainfall, E: evapotranspiration, If: infiltration, R: recharge, RR, runoff (Hortonian runoff and runoff on saturated surface), SS: subsurface flow, Fp: deep groundwater flow. Dotted lines represent minimum and maximum seasonal levels of the water table. 


\section{Tableau 1}

- Bilan hydrologique pour les deux années de suivi à l'échelle du bassin versant (BV) ; la pluie $(P)$ et le débit (Q) sont également calculés pour les deux autres exutoires intermédiaires ( $A$ et $K$, cf. Figure 1 ). La recharge de la nappe $(R)$ est estimée par la méthode des fluctuations piézométriques pour deux valeurs possibles de la porosité efficace de l'aquifère $(n=1 \%, n=5 \%)$. L'évapotranspiration réelle (ETR) est déduite par soustraction aux termes du bilan externe du bassin (P-Q), en négligeant le stockage interannuel dans l'aquifère (absence de tendance piézométrique) et les prélèvements anthropiques $\left(0,2 \mathrm{~mm} \mathrm{an}^{-1}\right)$.

\section{Table 1}

- Water balance for the two surveyed years at the basin scale (BV); rainfall $(\mathrm{P})$ and outflow (Q) are also reported for the two intermediate outlets (A and K, cf. Figure 1). Groundwater recharge (R) is estimated by the water table fluctuation method for two possible values of the specific yield $(n=1 \%, n$ $=5 \%)$. Effective evapotranspiration $(E T R)$ is deducted from the external terms of the balance (P-Q), considering no groundwater storage at the interannual scale and neglecting the human water consumption $\left(0.2 \mathrm{~mm} \mathrm{yr}^{-1}\right)$.

\begin{tabular}{|c|c|c|c|c|c|c|c|c|c|}
\hline & \multicolumn{3}{|c|}{$\mathrm{P} \mathbf{~ m m}$} & \multicolumn{3}{|c|}{ Q mm, [Q/P] \% } & \multicolumn{2}{|c|}{$\mathrm{R}$ mm, [R/P] \% } & \multirow{2}{*}{$\begin{array}{c}\text { ETR mm } \\
\text { BV }\end{array}$} \\
\hline & A & $\mathrm{K}$ & BV & A & $\mathrm{K}$ & BV & $\mathrm{BV}, n=1 \%$ & $\mathrm{BV}, n=5 \%$ & \\
\hline 2003 & 1634 & 1580 & 1514 & $\begin{array}{c}480 \\
{[29.4]}\end{array}$ & $\begin{array}{c}437 \\
{[27.7]}\end{array}$ & $\begin{array}{c}429 \\
{[28.3]}\end{array}$ & $\begin{array}{c}74 \\
{[4.9]}\end{array}$ & $\begin{array}{c}370 \\
{[24.4]}\end{array}$ & 1085 \\
\hline 2004 & 1066 & 1095 & 1140 & $\begin{array}{c}158 \\
{[14.8]}\end{array}$ & $\begin{array}{c}106 \\
{[9.6]}\end{array}$ & $\begin{array}{c}156 \\
{[13.7]}\end{array}$ & $\begin{array}{c}53 \\
{[4.6]}\end{array}$ & $\begin{array}{c}265 \\
{[23.2]}\end{array}$ & 984 \\
\hline
\end{tabular}

\section{Tableau 1}




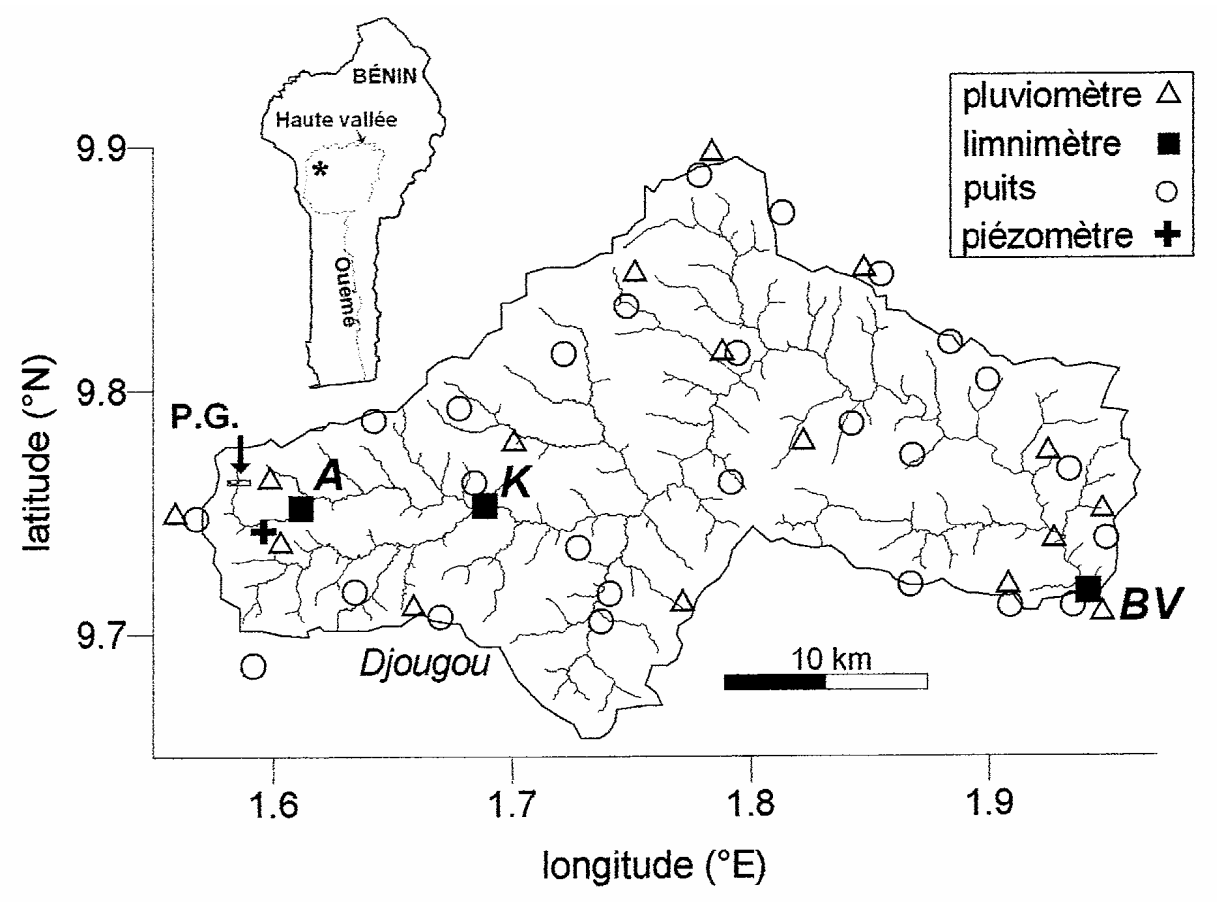

Figure 1 
Ouest

Est

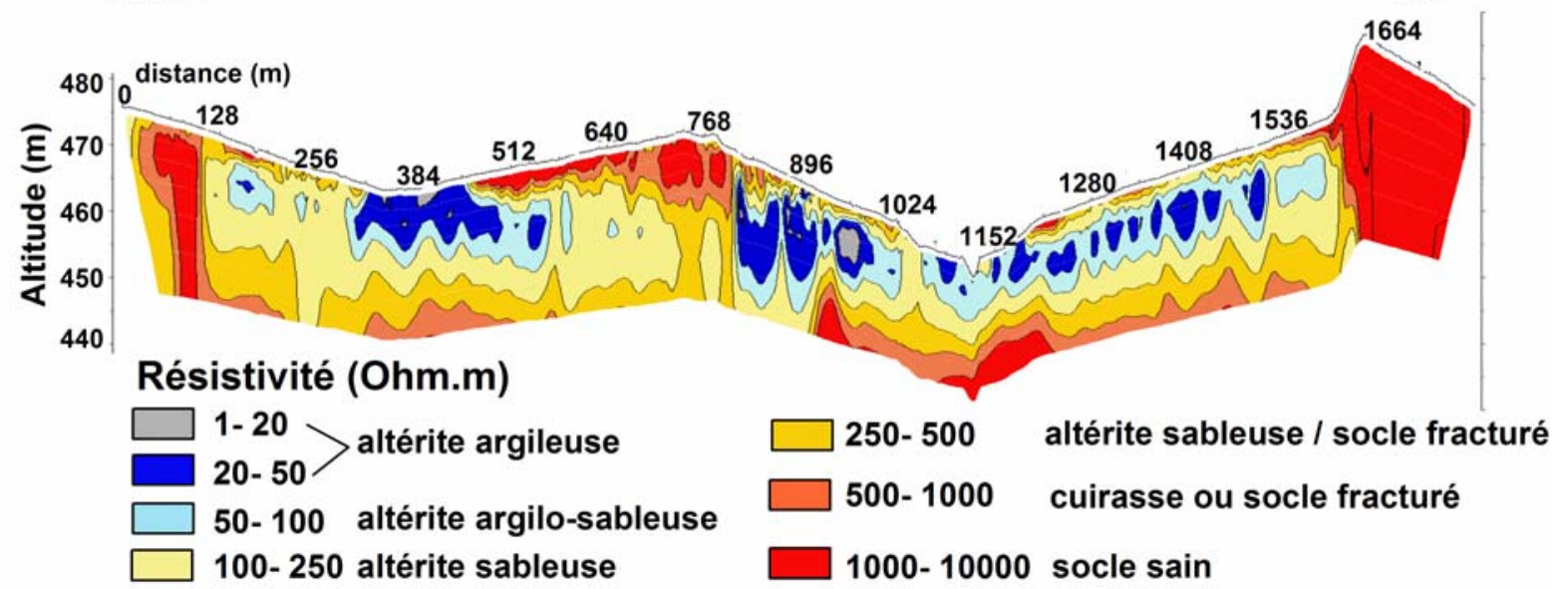

Figure 2 


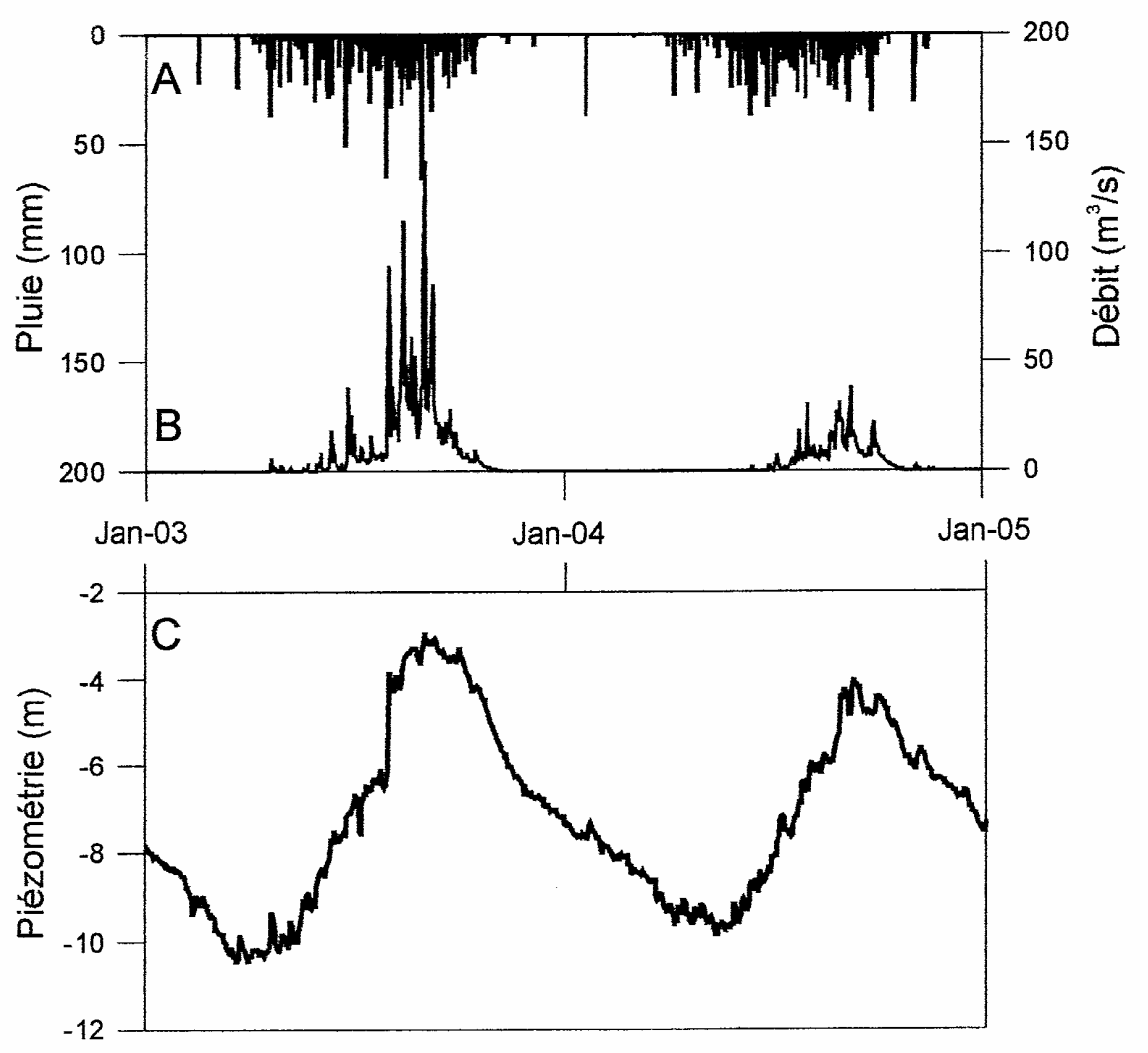

Figure 3 


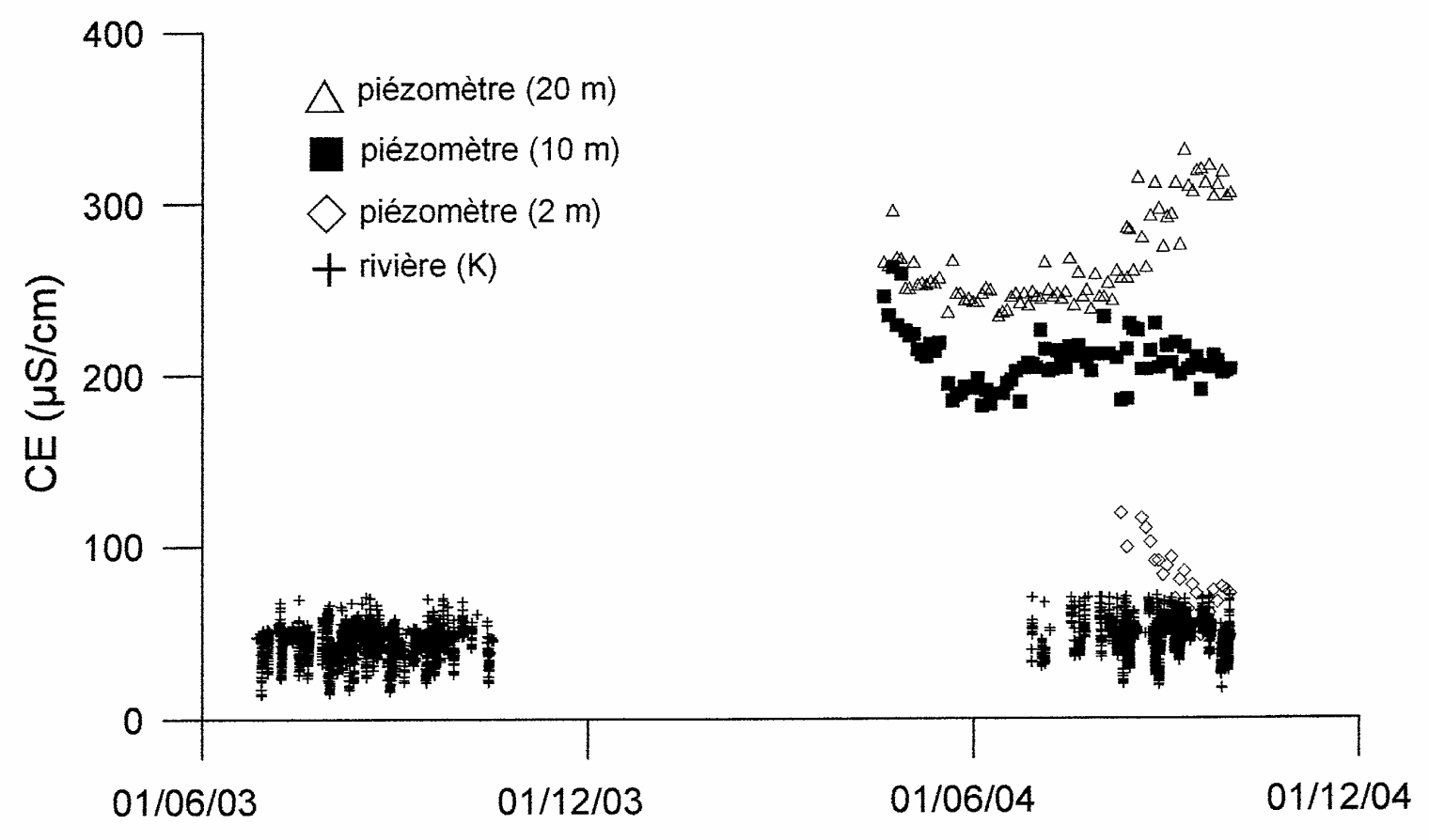

Figure 4 

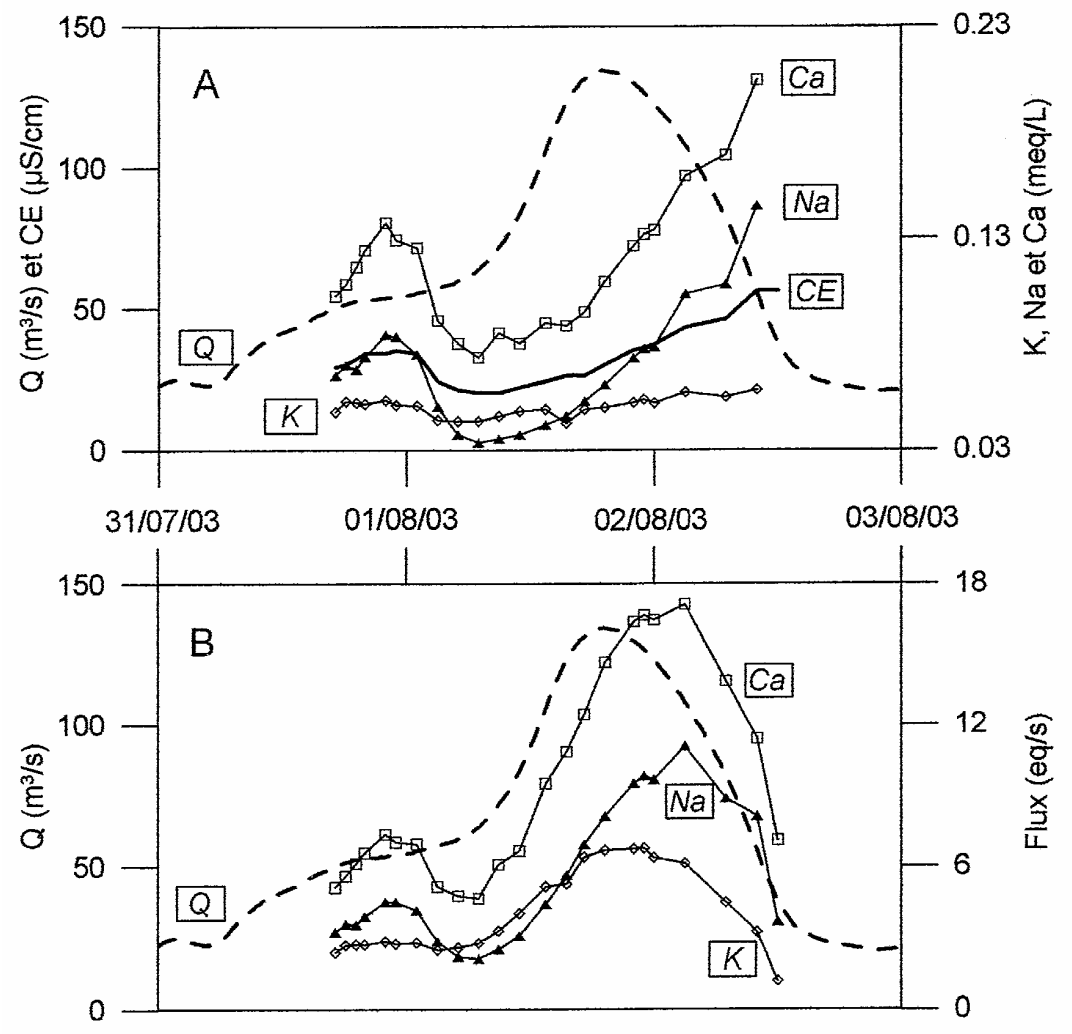

Figure 5 


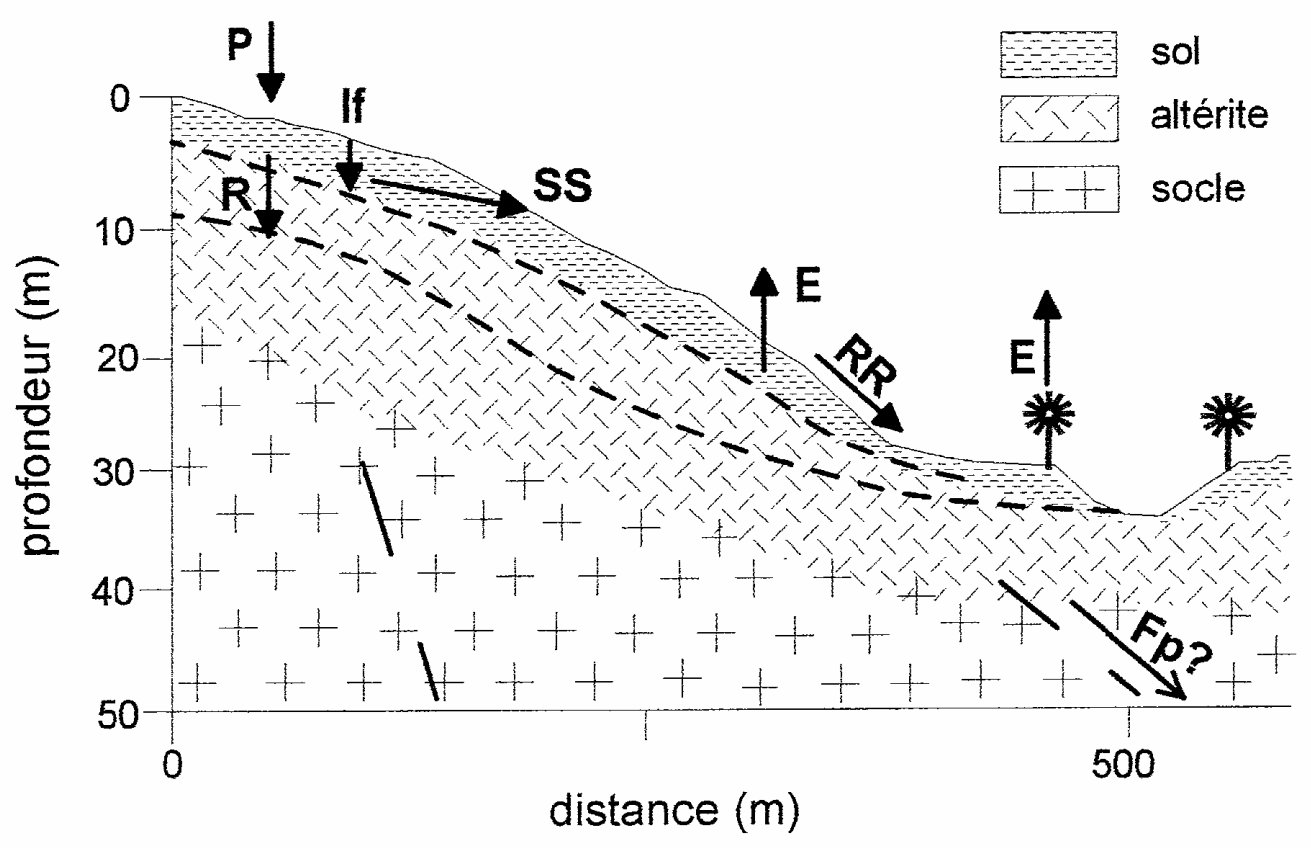

Figure 6 